\title{
Enumeration and Wilf-classification of permutations avoiding five patterns of length 4
}

\author{
Toufik Mansour* \\ Department of Mathematics, University of Haifa, 3498838 Haifa, Israel
}

(Received: 1 June 2020. Received in revised form: 10 July 2020. Accepted: 13 July 2020. Published online: 18 July 2020.)

(c) 2020 the author. This is an open access article under the CC BY (International 4.0) license (www. creativecommons.org/licenses/by/4.0/).

\begin{abstract}
Let $\mathcal{S}_{n}$ be the symmetric group of all permutations of $n$ letters. We show that there are exactly 3441 distinct Wilf classes for the permutations avoiding five patterns of length 4 . Moreover, for each $T \subset \mathcal{S}_{4}$ with $\# T=5$, we determine the generating function for the number of permutations in $\mathcal{S}_{n}(T)$, the set of all permutations of length $n$ that avoid each pattern in $T$.
\end{abstract}

Keywords: pattern avoidance; Wilf-equivalence; generating functions.

2020 Mathematics Subject Classification: 05A05, 05A15.

\section{Introduction}

A permutation on the set $[n]=\{1,2, \ldots, n\}$ is any arrangement of the elements of $[n]$. We denote the set of all permutations on $[n]$ by $\mathcal{S}_{n}$. For two permutations $\tau=\tau_{1} \tau_{2} \cdots \tau_{k} \in \mathcal{S}_{k}$ and $\sigma=\sigma_{1} \sigma_{2} \cdots \sigma_{n} \in \mathcal{S}_{n}$, we say that $\tau$ occurs as a pattern in $\sigma$ if there exists a subsequence in $\sigma$ which is order-isomorphic to $\tau$, that is, there exist $k$ indices $1 \leq i_{1}<i_{2}<\cdots<i_{k} \leq n$ such that $\sigma_{i_{a}}<\sigma_{i_{b}}$ if and only if $\tau_{a}<\tau_{b}$ for all $1 \leq a, b \leq k$. Otherwise, we say that $\sigma$ avoids $\tau$. We denote the set of all permutations in $\mathcal{S}_{n}$ that avoid $\tau$ by $\mathcal{S}_{n}(\tau)$. More generally, we define $\mathcal{S}_{n}(T)=\cap_{\tau \in T} \mathcal{S}_{n}(\tau)$, for a set $T$ of patterns. For example the permutation $543216987 \in \mathcal{S}_{9}$ avoids each of the patterns 1234 and 1324 but contains the pattern 2134. The research of pattern avoidance has received a lot of attention in the last couple of decades. Initial results were obtained by Knuth [9] and Simion and Schmidt [24] enumerated the permutations in $\mathcal{S}_{n}$ that avoid any subset of $\mathcal{S}_{3}$. In particular, Knuth [9] showed that

$$
\# \mathcal{S}_{n}(\tau)=C_{n}=\frac{1}{n+1}\left(\begin{array}{c}
2 n \\
n
\end{array}\right), \quad \tau \in \mathcal{S}_{3},
$$

where $C_{n}$ denotes the Catalan numbers. Note that the generating function for the Catalan numbers is given by $C(x)=$ $\sum_{n>0} C_{n} x^{n}=\frac{1-\sqrt{1-4 x}}{2 x}$ and it satisfies the equation $C(x)=1+x C^{2}(x)$.

Let $T$ and $T^{\prime}$ be any two sets of patterns. We say that $T$ and $T^{\prime}$ belong to the same symmetry class if and only if $T^{\prime}$ can be obtained from $T$ by the action of the dihedral group of order eight - generated by the symmetric operations - reverse, complement and inverse - on $T$. Moreover, we say that $T$ and $T^{\prime}$ belong to the same Wilf class if and only if $\# \mathcal{S}_{n}(T)=\# \mathcal{S}_{n}\left(T^{\prime}\right)$ for all $n \geq 0$.

We denote the number of distinct Wilf classes for the permutations avoiding exactly $k$ distinct patterns from $\mathcal{S}_{4}$ by $w_{k}$. In $[26,27,31]$ is shown that

$$
w_{1}=3 .
$$

Bóna [3] found an explicit formula for the generating function for the number of permutations in $\mathcal{S}_{n}(1342)$, and Regev [23] (see also, [7, 8]) found an explicit formula for the generating function for the number of permutations in $\mathcal{S}_{n}(1234)$. The enumeration of the remaining Wilf class, namely $\mathcal{S}_{n}(1324)$, is still an important open problem in the field of permutation patterns.

For pairs of patterns in $\mathcal{S}_{4}$, Le [12] established that

$$
w_{2}=38
$$

Vatter [29] showed that 12 of the 38 Wilf classes can be enumerated with so-called regular insertion encoding algorithm (the INSENC algorithm). This algorithm computes the (necessarily rational) generating function for any regular class, namely a class of permutations avoiding a set $T$ of patterns that has a regular insertion encoding (see also [1]). Some of these generating functions were computed by hand by Kremer and Shiu [10]. Miner [21] computed the generating functions for the classes $\mathcal{S}_{n}(\{4123,1324\}), \mathcal{S}_{n}(\{4123,1243\})$, and $\mathcal{S}_{n}(\{4123,1342\})$. Miner and Pantone [22] studied the classes 
$\mathcal{S}_{n}(\{2413,3412\})$ and $\mathcal{S}_{n}(\{3412,4123\})$. For details on the enumeration of each 38 Wilf classes, we refer the reader to Sequences A006317-A006318, A029759, A032351, A047849, A053617, A109033, A116704-10, A164651, A165524-A165646, A206736 in [25].

Triples of patterns from $\mathcal{S}_{4}$ were considered by Callan, Mansour and Shattuck [4,5] (see also [6]), where they showed that

$$
w_{3}=242 .
$$

Moreover, they found an explicit formula for the generating function for the number of permutations in $S_{n}(T)$ with $T \subset S_{4}$ and $\# T=3$ expect for only one case, where it is conjectured that the respective generating function is not differentially algebraic (see [1] and [25, Sequence A257562]).

Later, Mansour and Schork [14-16] showed that

$$
\begin{aligned}
& w_{6}=8438, \quad w_{7}=15392, \quad w_{8}=19002, \quad w_{9}=16293, \quad w_{10}=10624, \quad w_{11}=5857, \\
& w_{12}=3044, \quad w_{13}=1546, \quad w_{14}=786, \quad w_{15}=393, \quad w_{16}=198, \quad w_{17}=105, \\
& w_{18}=55, \quad w_{19}=28, \quad w_{20}=14, \quad w_{21}=8, \quad w_{22}=4, \quad w_{23}=2 \\
& w_{24}=1 \text {. }
\end{aligned}
$$

To compute $w_{k}$ for $k=3$ and $6 \leq k \leq 24$ several software programs were used:

- Firstly, they used the software of Kuszmaul [11] to generate all symmetry classes of $k$ patterns in $\mathcal{S}_{4}$ and the sequence $\left(\# \mathcal{S}_{n}(T)\right)_{n=1}^{16}$ for every symmetry class.

- Secondly, they used INSENC software (for example, see [29]) and they found (if possible) the generating function $F_{T}(x)=\sum_{n \geq 0} \# \mathcal{S}_{n}(T) x^{n}$ for all symmetry classes $T \subset \mathcal{S}_{4}$ with $\# T=3,6,7, \ldots, 24$. Those symmetry classes for which the generating function can be determined in this automatic way are called regular, while all other symmetry classes are called non-regular.

- Lastly, by hand they computed $w_{k}$ with $k=3,6,7, \ldots, 24$ by determining the generating functions $F_{T}(x)$ for all nonregular subsets $T$ with $\# T=k$.

Recently, by using this procedure, the author [13] showed that

$$
w_{4}=1100
$$

and determined the generating functions $F_{T}(x)$ for all non-regular subsets $T$ with $\# T=4$. Thus, it remains to find $w_{5}$. One of the aims of this paper is to show that

$$
w_{5}=3441,
$$

see Theorem 2.1.

\section{Enumeration and Wilf-classification of permutations avoiding five patterns}

Note that there are 42504 subsets of $\mathcal{S}_{4}$ with 5 permutations. By the software of Kuszmaul [11], we see that there are 5733 symmetry classes for such subsets. To find $w_{5}$, we use the following three steps. First, we determine the generating function $F_{T}(x)$, for each non-regular symmetry class $T$ with $\# T=5$. Second, by INSENC software, we find an explicit formula for the generating function $F_{T}(x)$, for all regular symmetry class $T$ with $\# T=5$, which determines 5156 . Therefore, we are left with 577 cases, that is, there are 577 non-regular symmetry classes in $\mathcal{S}_{4}$ with five patterns. We list them in Table 1 with the subset $T$ being marked by $*$ if the generating function $F_{T}(x)$ is not rational. After a tremendous work, we can formulate our main result.

Theorem 2.1. The generating functions $F_{T}(x)$ for non-regular symmetry classes $T \subset \mathcal{S}_{4}$ with $\# T=5$ are given in Table 1. In particular, we have $w_{5}=3441$, which shows that there are exactly 361 non-regular symmetry classes determined by rational generating functions and 216 non-regular symmetry classes determined by non-rational generating functions.

As a consequence of this theorem, we can state the following result.

Corollary 2.1. There are exactly 216 Wilf classes that are determined by an algebraic and non-rational generating function $F_{T}(x)$. 
Most of these algebraic non-rational generating functions are expressed in terms of the generating function $C(x)$ of Catalan numbers.

We comment that some cases in Theorem 2.1 depend on the enumeration of permutations avoiding a pattern in $\mathcal{S}_{3}$ and/or a pattern in $\mathcal{S}_{4}$. We refer the reader to [30] for a survey of these results, and also to the references [2,18-20,28,32] for other results in this direction.

For the sake of the reader, we have decided not to present most of the details of the proof of the main result but only explain the methods used to determine the 577 non-regular symmetry classes as stated in Theorem 2.1. For each we only provide one example to clarify the methods, for other examples, we refer the reader to $[4,5,13,17]$.

Left-Right-Maxima: A permutation $\pi$ expressed as $\pi=i_{1} \pi^{(1)} i_{2} \pi^{(2)} \cdots i_{m} \pi^{(m)}$ with $i_{1}<i_{2}<\cdots<i_{m}$ and $i_{j}>\max \left(\pi^{(j)}\right)$ for $1 \leq j \leq m$ is said to have $m$ left-right maxima (at $i_{1}, i_{2}, \ldots, i_{m}$ ). For a given set of patterns $T$, we define $\mathcal{L R M}_{n, m}(T)$ to be the set of all permutations in $\mathcal{S}_{n}(T)$ having exactly $m$ left-right-maxima. In order to find an explicit formula for the generating function $F_{T}(x)$, we determine the generating function $F_{T}(x ; m)=\sum_{n \geq m} \# \mathcal{L} \mathcal{R} \mathcal{M}_{n, m}(T) x^{n}$, for all $m \geq 1$ (clearly, the generating function in the case $m=0$ is given by 1 ), for more examples see [4-6].

Example 2.1. Let $T=\{3142,2143,1432,1342,1243\}$, namely, Case 3215 in Table 1. Let $\pi=i_{1} \pi^{(1)} i_{2} \pi^{(2)} \cdots i_{m} \pi^{(m)} \in$ $\mathcal{L} \mathcal{R} \mathcal{M}_{n, m}(T)$. Clearly, $F_{T}(x ; 1)=x F_{T}(x)$. If $m=2$, then $\pi$ can be written as either $\pi=(n-1) \pi^{(1)} n \pi^{(2)}$ with $\pi^{(1)}>\pi^{(2)}$ (we write $\alpha>\beta$ if each letter in $\alpha$ is greater than each letter of $\beta$ ), or $\pi=i n \alpha^{(i+1)}(i+1) \cdots \alpha^{(n-1)}(n-1) \alpha^{(n)}$ with $i>\alpha^{(i+1)}>\cdots>\alpha^{(n)}$ and $1 \leq i \leq n-2$ : this decomposition can be illustrated as
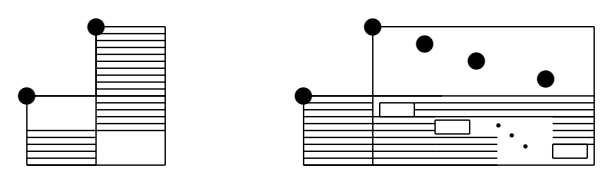

Thus,

$$
F_{T}(x ; 2)=x^{2} F_{T}^{2}(x)+\frac{x^{3} F_{T}^{2}(x)}{1-x F_{T}(x)} .
$$

For $m \geq 3, \pi$ can be written as $i_{1} \pi^{(1)} i_{2} \pi^{(2)} \cdots i_{m} \pi^{(m)}$ with $i_{1}>\pi^{(1)}>\cdots>\pi^{(m)}$, or $i_{1} i_{2} \alpha^{\left(i_{1}+1\right)}\left(i_{1}+1\right) \cdots \alpha^{\left(i_{2}-1\right)}\left(i_{2}+\right.$ 1) $\alpha^{\left(i_{2}\right)} i_{2} \pi^{(3)} i_{3} \cdots i_{m} \pi^{(m)}$ with $i_{2}-1>i_{1}>\alpha^{\left(i_{1}+1\right)}>\cdots>\alpha^{\left(i_{2}\right)}>\pi^{(3)}>\cdots>\pi^{(m)}$. Therefore,

$$
F_{T}(x ; m)=x^{m} F_{T}^{m}(x)+\frac{x^{m+1} F_{T}^{m}(x)}{1-x F_{T}(x)} .
$$

By summing over all contributions $F_{T}(x ; m)$, we obtain

$$
\begin{aligned}
F_{T}(x) & =1+x F_{T}(x)+x^{2} F_{T}^{2}(x)+\frac{x^{3} F_{T}^{2}(x)}{1-x F_{T}(x)}+\sum_{m \geq 3}\left(x^{m} F_{T}^{m}(x)+\frac{x^{m+1} F_{T}^{m}(x)}{1-x F_{T}(x)}\right) \\
& =\frac{1}{1-x F_{T}(x)}+\frac{x^{3} F_{T}^{2}(x)}{\left(1-x F_{T}(x)\right)^{2}} .
\end{aligned}
$$

By solving for $F_{T}(x)$, we obtain

$$
\begin{aligned}
F_{T}(x) & =\frac{x^{2}+2}{3 x} \\
& -\frac{(1-\sqrt{3} I) \sqrt[3]{2 x^{6}+12 x^{4}-9 x^{3}+15 x^{2}+9 x-2+3 \sqrt{3} x \sqrt{4 x^{5}-x^{4}+22 x^{3}-23 x^{2}+26 x-5}}}{6 x} \\
& -\frac{(1+\sqrt{3} I)\left(x^{4}+4 x^{2}-3 x+1\right)}{6 x \sqrt[3]{2 x^{6}+12 x^{4}-9 x^{3}+15 x^{2}+9 x-2+3 \sqrt{3} x \sqrt{4 x^{5}-x^{4}+22 x^{3}-23 x^{2}+26 x-5}}} \\
& =1+x+2 x^{2}+6 x^{3}+19 x^{4}+63 x^{5}+218 x^{6}+779 x^{7}+2852 x^{8}+10642 x^{9}+40325 x^{10}+\cdots,
\end{aligned}
$$

where $I^{2}=-1$.

The $\tau$-reduction argument: Suppose we have a set of patterns $T$ and a pattern $\tau \in \mathcal{S}_{3}$ such that each pattern $\sigma \in T$ contains $\tau$. Then, to determine the generating function $F_{T}(x)$, it is worth considering all the permutations in $\mathcal{S}_{n}(T)$ that either avoid $\tau$ or contain $\tau$. Thus, by (1) we have that

$$
F_{T}(x)=F_{\{\tau\}}(x)+F_{T ; \tau}(x)=C(x)+F_{T ; \tau}(x),
$$

where $F_{T ; \tau}(x)=\sum_{n \geq 0} \# \mathcal{S}_{n}(T ; \tau) x^{n}$ is the generating function for the number of permutations in $\mathcal{S}_{n}(T)$ that contain the pattern $\tau$. For several examples, see [17]. In particular, in [17] are determined cases 1274, 1312, 1992, 2238, 2254, 2542, 2799, 2822, 3401 and 3441 as shown in Table 1. 
Example 2.2. Let $T=T_{1307,1}$ in Table 1, namely $T=\{2431,2143,3142,4132,1324\}$. Let $\tau=132$. Then by $\tau$-reduction argument we have that

$$
F_{T}(x)=C(x)+G(x),
$$

where $G(x)$ is the generating function for the number of permutations in $\mathcal{S}_{n}(T ; \tau)$. By representing the permutations $\pi=$ $\pi_{1} \pi_{2} \cdots \pi_{n}$ in $\mathcal{S}_{n}(T ; \tau)$ as $\left\{\left(i, \pi_{i}\right) \mid i=1,2, \ldots, n\right\}$ in $\mathbb{N}^{2}$, we obtain the following decomposition

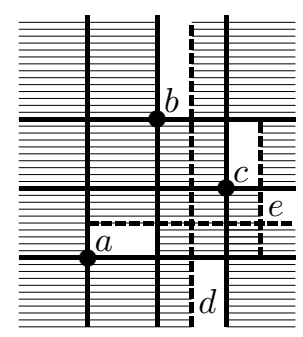

where the dots $\left(i, \pi_{i}\right)$ lie only on the cells $a, b, c, d$, . Here, each cell $a, b, c$ forms an increasing sequence. If the cell $d$ is not empty, then the cell e is empty (otherwise, the permutation contains 2143) and d avoids 132, so we have a contribution of $\frac{x^{3}(C(x)-1)}{(1-x)^{3}}$. Otherwise, the cell $d$ is empty and e avoids 213 and 132, which gives a contribution of $\frac{x^{3}}{(1-x)^{2}(1-2 x)}$. Hence,

$$
\sum_{n \geq 0} \# \mathcal{S}_{n}(T ; \tau) x^{n}=\frac{x^{3}(C(x)-1)}{(1-x)^{3}}+\frac{x^{3}}{(1-x)^{2}(1-2 x)}=\frac{x^{3} C(x)}{(1-x)^{3}}+\frac{x^{4}}{(1-x)^{3}(1-2 x)},
$$

which leads to

$$
F_{T}(x)=C(x)+\frac{x^{3} C(x)}{(1-x)^{3}}+\frac{x^{4}}{(1-x)^{3}(1-2 x)} .
$$

\begin{tabular}{|c|c|c|c|}
\hline \multicolumn{4}{|r|}{ Begin of Table 1} \\
\hline$i$ & $j$ & $T=T_{i j}$ & $F_{T}(x)$ \\
\hline 512 & 17 & $\{2143,3142,3124,1324,1423\}$ & $(1-2 x)(1-x)^{2} /\left(1-5 x+8 x^{2}-6 x^{3}+x^{4}\right)$ \\
\hline \multirow[t]{4}{*}{528} & 2 & $\{2341,3412,1324,4123,1423\}$ & \\
\hline & 3 & $\{2314,3412,1342,1324,4123\}$ & \\
\hline & 4 & $\{2314,3412,1342,4123,1234\}$ & \\
\hline & 5 & $\{2134,3412,1423,1243,1234\}$ & $\left(1-7 x+21 x^{2}-33 x^{3}+30 x^{4}-17 x^{5}+4 x^{6}\right) /\left((1-2 x)(1-x)^{6}\right)$ \\
\hline \multirow[t]{4}{*}{545} & 4 & $\{2341,3412,4123,1423,1243\}$ & \\
\hline & 5 & $\{2341,3412,4123,1423,1234\}$ & \\
\hline & 7 & $\{3412,3124,1324,1243,1234\}$ & \\
\hline & 8 & $\{3412,1342,4123,1243,1234\}$ & $\left(1-6 x+15 x^{2}-18 x^{3}+12 x^{4}-3 x^{5}\right) /\left((1-2 x)(1-x)^{5}\right)$ \\
\hline \multirow[t]{2}{*}{564} & 11 & $\{2413,2143,4312,3142,1324\}$ & \\
\hline & 19 & $\{2134,3412,3142,4123,1423\}$ & $\left(1-6 x+14 x^{2}-14 x^{3}+6 x^{4}-2 x^{5}\right) /\left((1-2 x)^{2}(1-x)^{3}\right)$ \\
\hline \multirow[t]{3}{*}{596} & 3 & $\{4231,3412,3142,1342,1324\}$ & \\
\hline & 10 & $\{4213,4312,4132,1324,1423\}$ & \\
\hline & 18 & $\{4312,3412,1324,4123,1423\}$ & $\left(1-5 x+9 x^{2}-5 x^{3}+x^{4}\right) /\left((1-2 x)^{2}(1-x)^{2}\right)$ \\
\hline 612 & 2 & $\{4231,3412,1342,1324,1423\}$ & $\left(1-4 x+6 x^{2}-2 x^{3}+2 x^{4}\right) /\left((1-2 x)(1-x)^{3}\right)$ \\
\hline \multirow[t]{12}{*}{613} & 5 & $\{2341,3412,3124,1342,4123\}$ & \\
\hline & 9 & $\{2314,3412,3124,1342,1423\}$ & \\
\hline & 10 & $\{2314,3412,1342,4123,1423\}$ & \\
\hline & 14 & $\{2134,3412,3142,1324,1423\}$ & \\
\hline & 15 & $\{2134,3412,3124,1342,4123\}$ & \\
\hline & 16 & $\{2134,3412,3124,1342,1423\}$ & \\
\hline & 17 & $\{2134,3412,1342,4123,1234\}$ & \\
\hline & 20 & $\{3412,3124,1342,4123,1234\}$ & \\
\hline & 21 & $\{3412,3124,1342,1423,1243\}$ & \\
\hline & 22 & $\{3412,3124,1342,1423,1234\}$ & \\
\hline & 23 & $\{3412,3124,1342,1243,1234\}$ & \\
\hline & 25 & $\{3412,1342,1324,4123,1234\}$ & $\left(1-5 x+10 x^{2}-8 x^{3}+4 x^{4}\right) /\left((1-2 x)(1-x)^{4}\right)$ \\
\hline 630 & 1 & $\{4231,2413,3412,3142,1324\}$ & $\left(1-6 x+14 x^{2}-14 x^{3}+6 x^{4}-2 x^{5}+2 x^{6}\right) /\left((1-2 x)^{2}(1-x)^{3}\right)$ \\
\hline 633 & 1 & $\{4231,2413,3142,1324,4123\}$ & $\left(1-6 x+14 x^{2}-14 x^{3}+6 x^{4}-2 x^{5}+3 x^{6}\right) /\left((1-2 x)^{2}(1-x)^{3}\right)$ \\
\hline 634 & 1 & $\{4231,2413,3142,1324,1423\}$ & $\left(1-7 x+19 x^{2}-23 x^{3}+11 x^{4}-x^{5}+x^{6}\right) /\left((1-2 x)^{3}(1-x)^{2}\right)$ \\
\hline 635 & 1 & $\{4231,2413,3142,1324,1243\}$ & $\left(1-10 x+44 x^{2}-110 x^{3}+171 x^{4}-174 x^{5}+124 x^{6}-69 x^{7}+29 x^{8}-5 x^{9}\right) /\left((1-x)^{7}(1-2 x)^{2}\right)$ \\
\hline \multirow[t]{2}{*}{643} & 2 & $\{4231,2413,4132,1342,1324\}$ & \\
\hline & 3 & $\{4231,3142,1324,4123,1423\}$ & $\left(1-6 x+14 x^{2}-14 x^{3}+6 x^{4}-x^{5}+x^{6}\right) /\left((1-x)^{3}(1-2 x)^{2}\right)$ \\
\hline \multirow[t]{3}{*}{674} & 1 & $\{4231,2143,3412,3142,1324\}$ & \\
\hline & 4 & $\{4213,2143,3412,4132,1324\}$ & \\
\hline & 6 & $\{2143,4312,3142,1324,4123\}$ & $\left(1-5 x+10 x^{2}-8 x^{3}+4 x^{4}-4 x^{5}\right) /\left((1-x)^{4}(1-2 x)\right)$ \\
\hline \multirow[t]{2}{*}{678} & 1 & $\{4231,2143,3412,1324,4123\}$ & \\
\hline & 2 & $\{4231,2143,3412,1324,1243\}$ & $\left(1-4 x+6 x^{2}-2 x^{3}+2 x^{4}-7 x^{5}-2 x^{6}\right) /\left((1-x)^{3}(1-2 x)\right)$ \\
\hline 679 & 1 & $\{4231,2143,3412,1324,1423\}$ & $\left(1-5 x+10 x^{2}-8 x^{3}+4 x^{4}-6 x^{5}+2 x^{6}\right) /\left((1-x)^{4}(1-2 x)\right)$ \\
\hline 686 & 1 & $\{4231,2143,3142,1324,1423\}$ & $\left(1-7 x+20 x^{2}-28 x^{3}+20 x^{4}-9 x^{5}+6 x^{6}-4 x^{7}\right) /\left((1-x)^{4}(1-2 x)^{2}\right)$ \\
\hline 696 & 1 & $\{4231,2143,3124,1324,1423\}$ & $\left(1-5 x+10 x^{2}-8 x^{3}+4 x^{4}-2 x^{5}+2 x^{7}\right) /\left((1-x)^{4}(1-2 x)\right)$ \\
\hline \multirow[t]{4}{*}{697} & 5 & $\{4213,2143,4312,4132,1324\}$ & \\
\hline & 6 & $\{2143,3412,3124,1324,1423\}$ & \\
\hline & 8 & $\{2134,3412,3124,1342,1243\}$ & \\
\hline & 9 & $\{2134,3412,1342,1423,1243\}$ & $\left(1-6 x+15 x^{2}-18 x^{3}+12 x^{4}-6 x^{5}\right) /\left((1-x)^{5}(1-2 x)\right)$ \\
\hline 711 & 1 & $\{4231,2143,1342,1324,1423\}$ & $\left(1-8 x+27 x^{2}-48 x^{3}+48 x^{4}-29 x^{5}+11 x^{6}+x^{7}-6 x^{8}+5 x^{9}-x^{1} 0\right) /\left((1-x)^{5}(1-2 x)^{2}\right)$ \\
\hline \multirow[t]{2}{*}{808} & $\begin{array}{l}1 \\
2\end{array}$ & $\begin{array}{l}\{4231,4312,3412,3142,1324\} \\
\{4231,3412,3142,1324,4123\}\end{array}$ & \\
\hline & 3 & $\{4231,3142,1432,1324,1243\}$ & $\left(1-8 x+27 x^{2}-48 x^{3}+48 x^{4}-30 x^{5}+17 x^{6}-12 x^{7}+6 x^{8}\right) /\left((1-x)^{5}(1-2 x)^{2}\right)$ \\
\hline \multirow[t]{2}{*}{809} & 1 & $\{4231,4312,3412,4132,1324\}$ & \\
\hline & 2 & $\{4231,3412,4132,1324,4123\}$ & $\left(1-7 x+20 x^{2}-28 x^{3}+20 x^{4}-9 x^{5}+2 x^{6}+3 x^{7}-x^{8}\right) /\left((1-x)^{4}(1-2 x)^{2}\right)$ \\
\hline 813 & 9 & $\{2143,3412,3142,1324,4123\}$ & $\left(1-6 x+15 x^{2}-18 x^{3}+12 x^{4}-7 x^{5}+2 x^{6}\right) /\left((1-x)^{5}(1-2 x)\right)$ \\
\hline 815 & 1 & $\{4231,4312,3412,1324,4123\}$ & $\left(1-6 x+14 x^{2}-14 x^{3}+6 x^{4}-3 x^{5}-x^{6}+4 x^{7}\right) /\left((1-x)^{3}(1-2 x)^{2}\right)$ \\
\hline \multirow[t]{2}{*}{816} & 1 & $\{4231,4312,3412,1324,1423\}$ & \\
\hline & 2 & $\{4231,3412,3142,1324,1423\}$ & $\left(1-6 x+14 x^{2}-14 x^{3}+6 x^{4}-3 x^{5}+x^{6}\right) /\left((1-x)^{3}(1-2 x)^{2}\right)$ \\
\hline
\end{tabular}

Table 1: Non-regular classes and their enumeration 


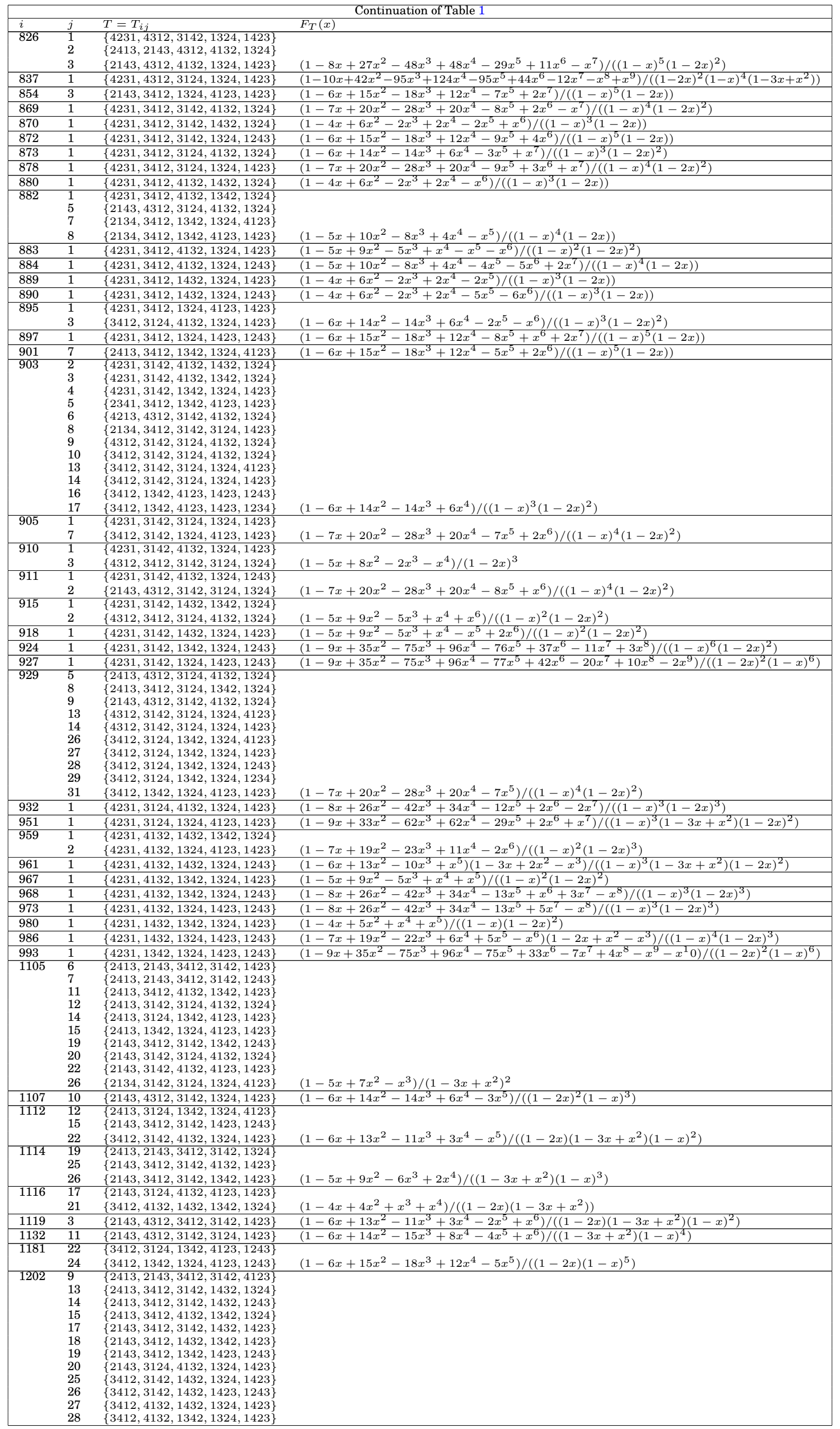




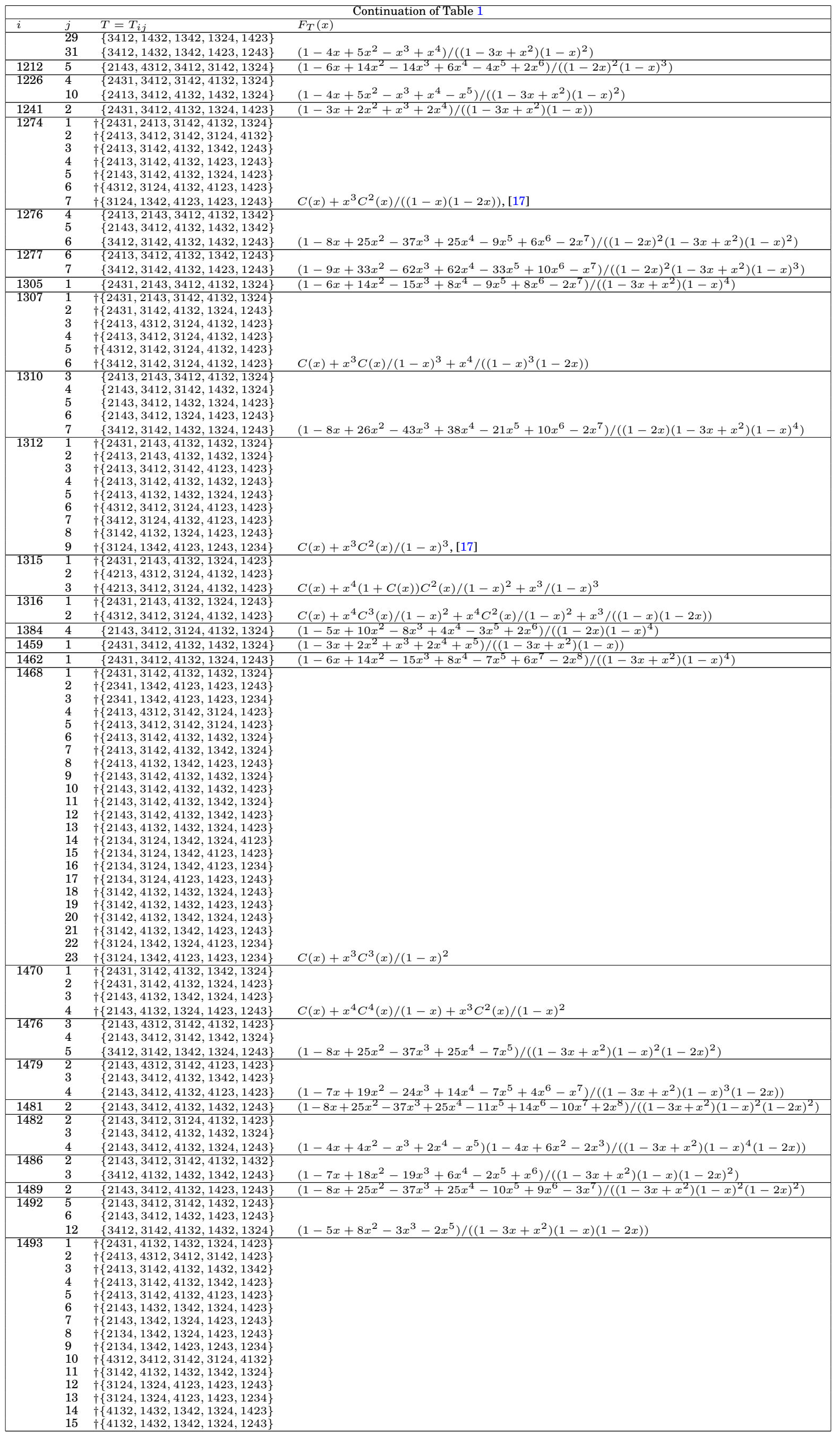




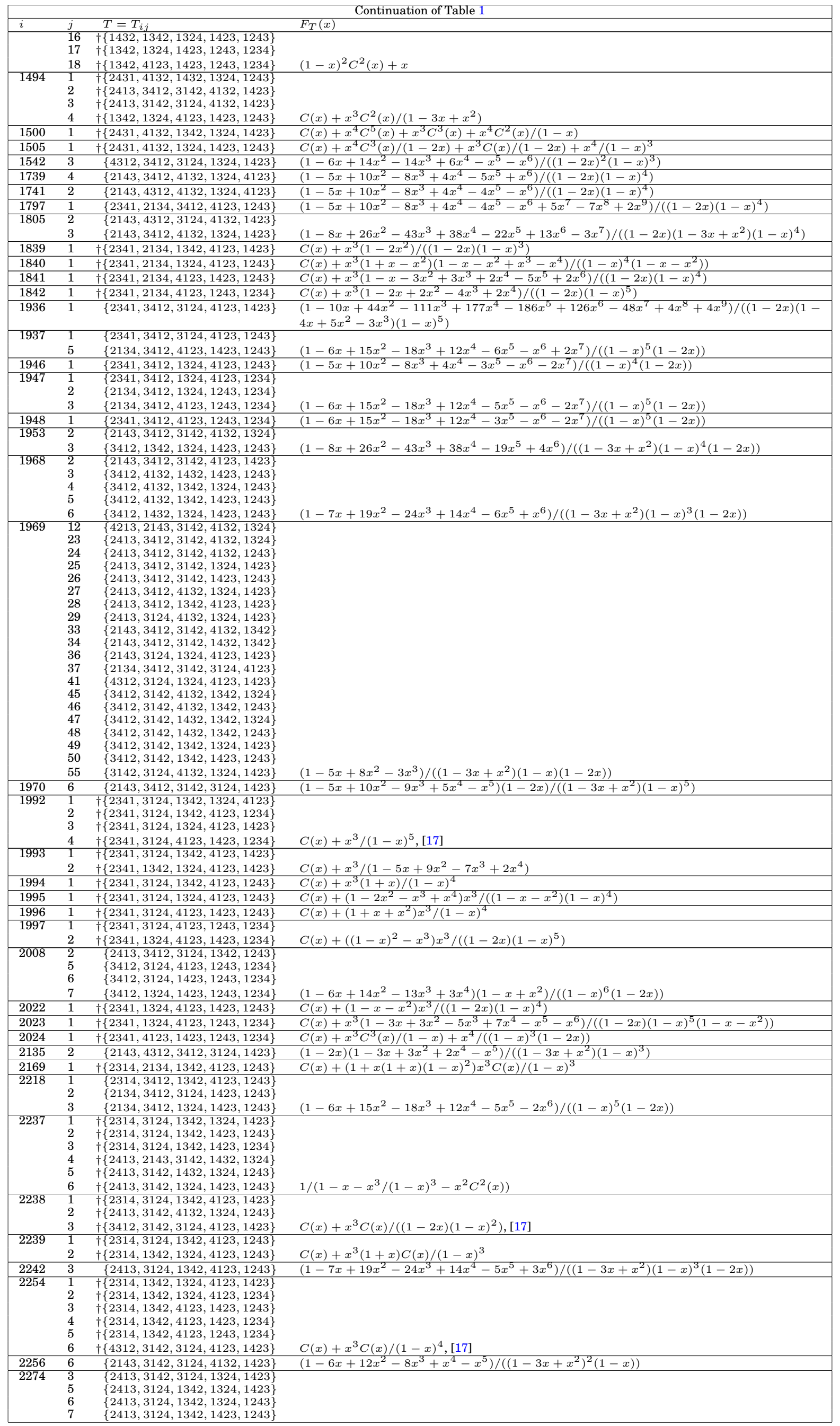




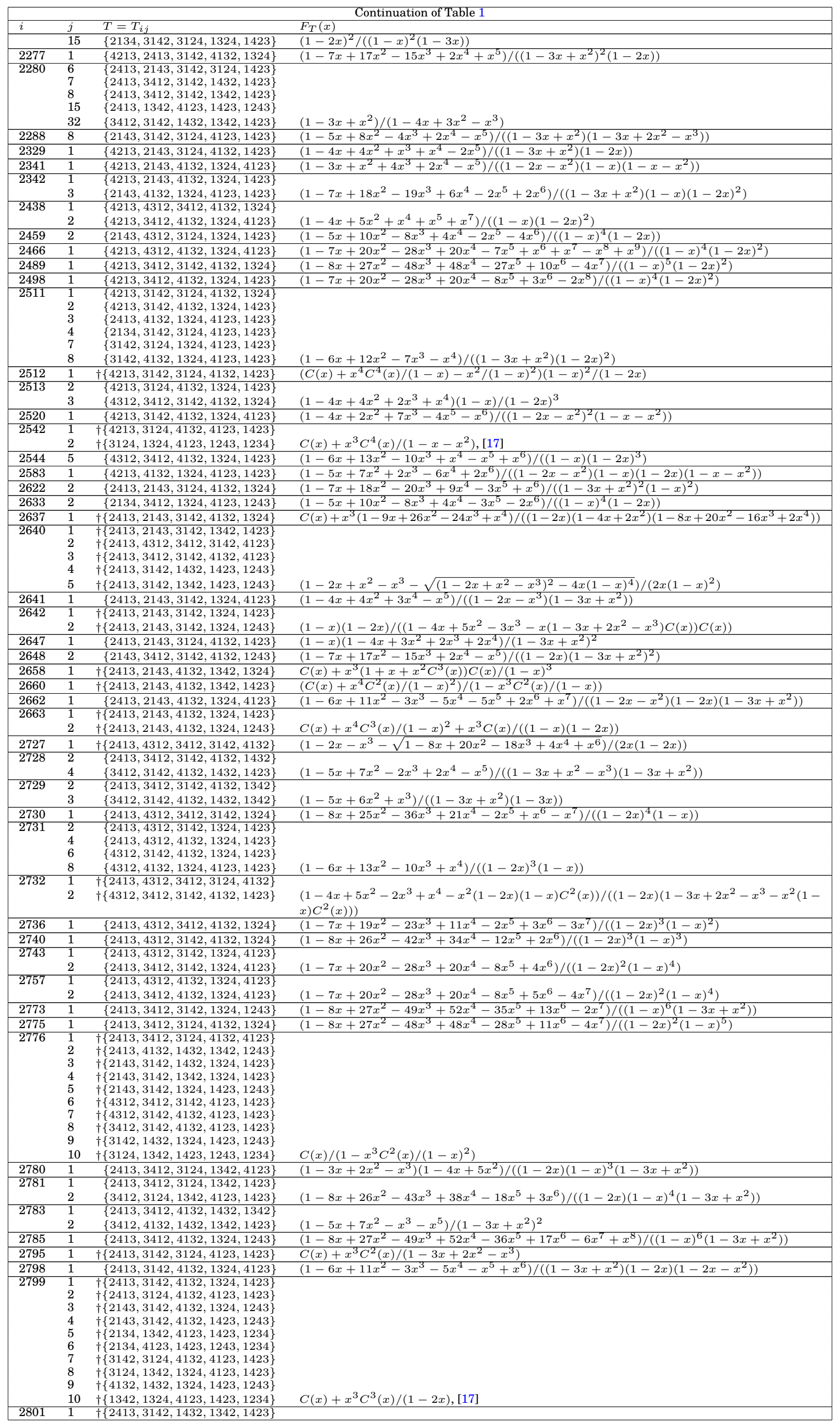




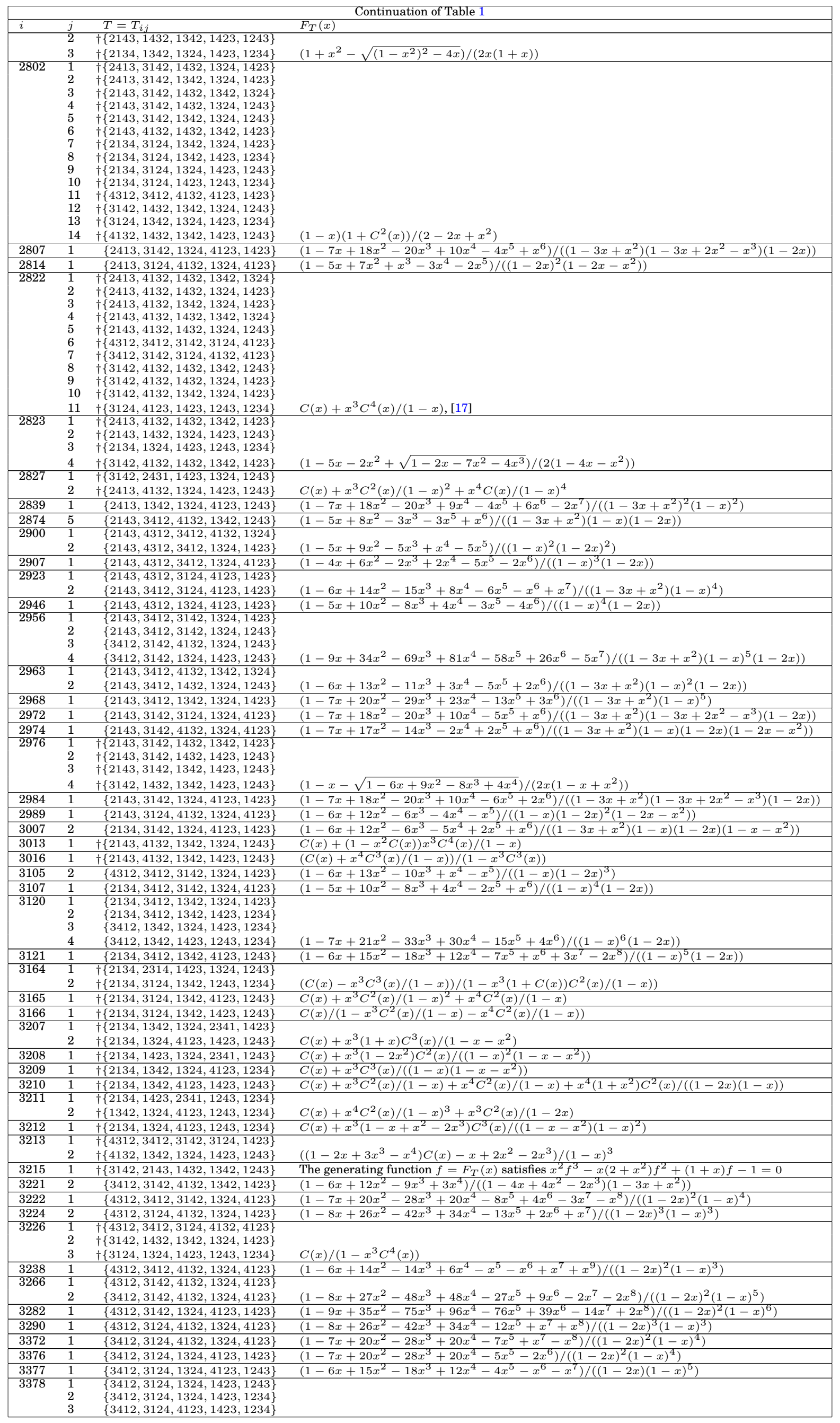




\begin{tabular}{|lllcl|}
\hline \multicolumn{4}{|c|}{ Continuation of Table 1 } \\
\hline$i$ & $j$ & $T=T_{i j}$ & $F_{T}(x)$ \\
\hline & 4 & $\{3412,1324,4123,1423,1243\}$ & \\
& 5 & $\{3412,1324,4123,1423,1234\}$ & $\left(1-7 x+21 x^{2}-33 x^{3}+30 x^{4}-15 x^{5}+2 x^{6}\right) /\left((1-2 x)(1-x)^{6}\right)$ \\
\hline 3379 & 1 & $\{3412,3124,4123,1423,1243\}$ & $\left(1-9 x+35 x^{2}-75 x^{3}+96 x^{4}-74 x^{5}+30 x^{6}-3 x^{7}\right) /\left((1-2 x)^{2}(1-x)^{6}\right)$ \\
\hline 3381 & 1 & $\{3412,4132,1432,1324,1243\}$ & $\left(1-8 x+26 x^{2}-43 x^{3}+38 x^{4}-21 x^{5}+9 x^{6}+x^{7}-x^{8}\right) /\left((1-2 x)(1-x)^{4}\left(1-3 x+x^{2}\right)\right)$ \\
\hline 3383 & 1 & $\{3412,4132,1324,4123,1423\}$ & $\left(1-8 x+27 x^{2}-48 x^{3}+48 x^{4}-27 x^{5}+8 x^{6}-2 x^{8}\right) /\left((1-2 x)^{2}(1-x)^{5}\right)$ \\
\hline 3385 & 1 & $\{3412,4132,1324,1423,1243\}$ & $\left(1-7 x+20 x^{2}-29 x^{3}+23 x^{4}-13 x^{5}+2 x^{6}+3 x^{7}-x^{8}\right) /\left((1-x)^{5}\left(1-3 x+x^{2}\right)\right)$ \\
\hline 3388 & 1 & $\{3412,1324,4123,1243,1234\}$ & $\left(1-6 x+15 x^{2}-18 x^{3}+12 x^{4}-4 x^{5}-x^{6}-2 x^{7}\right) /\left((1-2 x)(1-x)^{5}\right)$ \\
\hline 3389 & 1 & $\{3412,4123,1423,1243,1234\}$ & $\left(1-8 x+27 x^{2}-48 x^{3}+48 x^{4}-26 x^{5}+5 x^{6}-x^{7}\right) /\left((1-2 x)^{2}(1-x)^{5}\right)$ \\
\hline 3390 & 1 & $\{3142,3124,4132,1324,4123\}$ & $\left(1-4 x+3 x^{2}+4 x^{3}+x^{4}\right)(1-x) /\left((1-2 x)^{2}\left(1-2 x-x^{2}\right)\right)$ \\
\hline 3401 & 1 & $\dagger\{3142,1342,1324,1423,1243\}$ & \\
& 2 & $\dagger\{3124,1342,1324,1423,1243\}$ & $C(x) /\left(1-x^{3} C^{2}(x) /(1-2 x)\right),[17]$ \\
\hline 3407 & 1 & $\{3124,4132,1324,4123,1423\}$ & $\left(1-7 x+18 x^{2}-18 x^{3}+3 x^{4}+4 x^{5}\right) /(1-2 x)^{4}$ \\
\hline 3425 & 1 & $\dagger\{2314,1423,1324,2341,1243\}$ & $C(x)+x^{3} C^{2}(x) /(1-2 x)+x^{4} C^{2}(x) /(1-x)^{2}$ \\
\hline 3426 & 1 & $\dagger\{3124,1342,1324,1243,1234\}$ & $C(x) /\left(1-x^{3}(1-x)\left(1+C^{2}(x)\right) C^{3}(x) /\left(2-2 x+x^{2}\right)\right)$ \\
\hline 3441 & 1 & $\dagger\{1324,4123,1423,1243,1234\}$ & $C(x)+(1-x) x^{3} C^{4}(x) /(1-2 x),[17]$ \\
\hline & & & End of Table 1 \\
\hline
\end{tabular}

\section{Conclusion}

This paper completes the Wilf-classification problem for permutations avoiding a set of patterns in $\mathcal{S}_{4}$, that is, we determine all subsets $T, T^{\prime} \subset \mathcal{S}_{4}$ with $\# T=\# T^{\prime}=k$ where $\# \mathcal{S}_{n}(T)=\# \mathcal{S}_{n}\left(T^{\prime}\right)$ for all $n \geq 0$. Moreover, it completes finding an explicit formula for the generating function $F_{T}(x)$ for any $T \subseteq \mathcal{S}_{4}$, expect for only two cases:

- $F_{\{1324\}}(x)$,

- $F_{\{3214,1324,2134\}}(x)$,

as discussed in the introduction.

\section{Acknowledgment}

The author is grateful to the anonymous referees for careful reading of the manuscript.

\section{References}

[1] M. H. Albert, C. Homberger, J. Pantone, N. Shar, V. Vatter, Generating permutations with restricted containers, J. Combin. Theory Ser. A 157 (2018) 205-232.

[2] M. Atkinson, Restricted permutations, Discrete Math. 195 (1999) 27-38.

[3] M. Bóna, Exact enumeration of 1342-avoiding permutations: a close link with labeled trees and planar maps, J. Combin. Theory Ser. A 80 (1997) $257-272$.

[4] D. Callan, T. Mansour, M. Shattuck, Wilf classification of triples of 4-letter patterns I, Discrete Math. Theoret. Comput. Sci. 19 (2017) Art\# 5.

[5] D. Callan, T. Mansour, M. Shattuck, Wilf classification of triples of 4-letter patterns II, Discrete Math. Theoret. Comput. Sci. 19 (2017) Art\# 6.

[6] D. Callan, T. Mansour, M. Shattuck, Enumeration of permutations avoiding a triple of 4-letter patterns is almost all done, Pure Math. Appl. 28 (2019) 14-69.

[7] I. M. Gessel, Symmetric functions and P-recursiveness, J. Combin. Theory Ser. A 53 (1990) 257-285.

[8] D. Gouyou-Beauchamps, Standard Young tableaux of height 4 and 5, European J. Combin. 10 (1989) 69-82.

[9] D. E. Knuth, The Art of Computer Programming, 3rd Edition, Addison Wesley, Reading, 1997.

[10] D. Kremer, W. C. Shiu, Finite transition matrices for permutations avoiding pairs of length four patterns, Discrete Math. 268 (2003) 171-183.

[11] W. Kuszmaul, Fast algorithms for finding pattern avoiders and counting pattern occurrences in permutations, Math. Comp. 87 (2018) $987-1011$.

[12] I. Le, Wilf classes of pairs of permutations of length 4, Electron. J. Combin. 12 (2005) Art\# R25.

[13] T. Mansour, Enumeration and Wilf-classification of permutations avoiding four patterns of length 4, Discrete Math. Lett. 3 (2020) 67-94.

[14] T. Mansour, M. Schork, Wilf classification of subsets of four letter patterns, J. Combin. Number Theory 8 (2016) 1-129.

[15] T. Mansour, M. Schork, Wilf classification of subsets of eight and nine four-letter patterns, J. Combin. Number Theory 8 (2016) 257-283.

[16] T. Mansour, M. Schork, Wilf classification of subsets of six and seven four-letter patterns, J. Combin. Number Theory 9 (2017) 169-213.

[17] T. Mansour, M. Schork, Permutation patterns and cell decompositions, Math. Comput. Sci. 13 (2019) 169-183.

[18] T. Mansour, Z. Stankova, 321-polygon-avoiding permutations and Chebyshev polynomials, Electron. J. Combin. 9 (2003) Art\# R5.

[19] T. Mansour, A. Vainshtein, Layered restrictions and Chebyshev polynomials, Ann. Comb. 5 (2001) 451-458.

[20] T. Mansour, A. Vainshtein, Restricted 132-avoiding permutations, Adv. in Appl. Math. 26 (2001) 258-269.

[21] S. Miner, Enumeration of several two-by-four classes, arXiv:1610.01908 [math.CO].

[22] S. Miner, J. Pantone, Completing the structural analysis of the $2 \times 4$ permutation classes, arXiv:1802.00483 [math.CO].

[23] A. Regev, Asymptotic values for degrees associated with strips of Young diagrams, Adv. Math. 41 (1981) 115-136.

[24] R. Simion and F.W. Schmidt, Restricted permutations, European J. Combin. 6 (1985) 383-406.

[25] N. J. A. Sloane, The On-Line Encyclopedia of Integer Sequences, https ://oeis . org.

[26] Z. E. Stankova, Forbidden subsequences, Discrete Math. 132 (1994) 291-316.

[27] Z. E. Stankova, Classification of forbidden subsequences of length four, European J. Combin. 17 (1996) 501-517.

[28] V. Vatter, Finitely labeled generating trees and restricted permutations, J. Symbolic Comput. 41 (2006) 559-572.

[29] V. Vatter, Finding regular insertion encodings for permutation classes, J. Symbolic Comput. 47 (2012) 259-265.

[30] Wikipedia, Enumerations of Specific Permutation Classes, https://en.wikipedia.org/wiki/Enumerationsof specificpermutationclasses.

[31] J. West, Generating trees and the Catalan and Schröder numbers, Discrete Math. 146 (1995) 247-262.

[32] J. West, Generating trees and forbidden subsequences, Discrete Math. 157 (1996) 363-374. 